\title{
Diagnosis of follicular lymphoma of the small bowel by video capsule endoscopy
}

\author{
Juan Egea-Valenzuela, José Manuel Castillo-Espinosa, Antonio Sánchez-Torres, \\ Fernando Alberca-de-las-Parras and Fernando Carballo Álvarez
}

Department of Gastroenterology. Hospital Clínico Universitario Virgen de la Arrixaca. Murcia, Spain

\section{INTRODUCTION}

Follicular lymphoma is one of the most common types of non-Hodgkin lymphoma in western countries. It is usually a silent disease and gastrointestinal location is rare, including less than $4 \%$ of all non-Hodgkin lymphomas of the digestive tract. Endoscopy of the small bowel carried out with video capsule is useful for the diagnosis of this entity.

\section{CASE REPORT}

A 61 year-old asymptomatic male was included in the colorectal cancer screening programme. As fecal occult blood tests were positive, a colonoscopy was carried out, but the examination was not complete due to low tolerance to the procedure. Following the established protocol in our center a colonography by computed tomography (CT-colonography) was made. Colonic lesions were not observed but multiple mesenteric lymph nodes were diagnosed instead (Fig. 1). After this, a positron emission tomography (PET) described multiple abdominal nodes with enhanced metabolism. With the clinical suspicion of a lymphoproliferative process, we decided to carry out an endoscopic examination of the small bowel with video capsule in which we observed an amount of whitish nodules and small polipoid lessions (Fig. 2), located between the medium portion of jejunum and ileon. In the distal portions of the small bowel, the lesions were bigger, with thickened mucosal folds, and some of these nodules were ulcerated (Fig. 3). According to the reports in literature, these findings suggested a lymphoproliferative disorder of the small bowel (1). The specimens obtained by enteroscopy and CT-guided fine-needle aspiration confirmed the diagnosis of studium IIA follicular lymphoma.

\section{DISCUSSION}

Although gastrointestinal follicular lymphoma is rare, the digestive tract is the most frequent extranodal location for this type of non-Hodgkin lymphoma. Development of small bowel endoscopy, with the use of video capsule and the different modalities of enteroscopy, has brought to a better diagnosis of this entity, and a more efficient follow-up of these patients after chemotherapy (2-4). In scientific literature, we only find descriptions of isolated cases or short series reports, but these works have made possible to define the most common aspect of these lesions on the intestinal mucosa, and establish an endoscopic differential diagnosis with other entities as different types of enteritis and other kinds of lymphoma $(1,3,4)$.

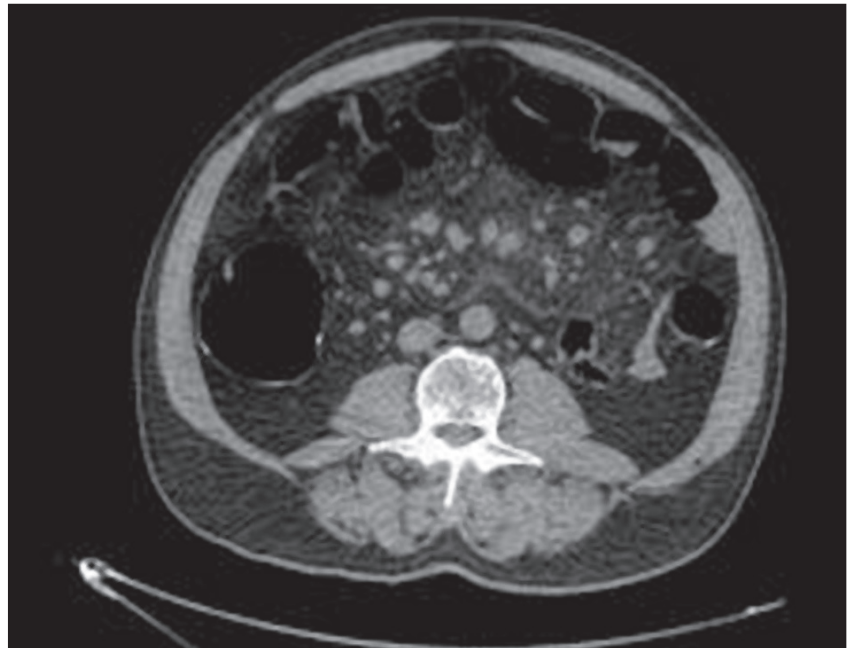

Fig. 1. Multiple mesenteric nodes seen in computed tomography. 


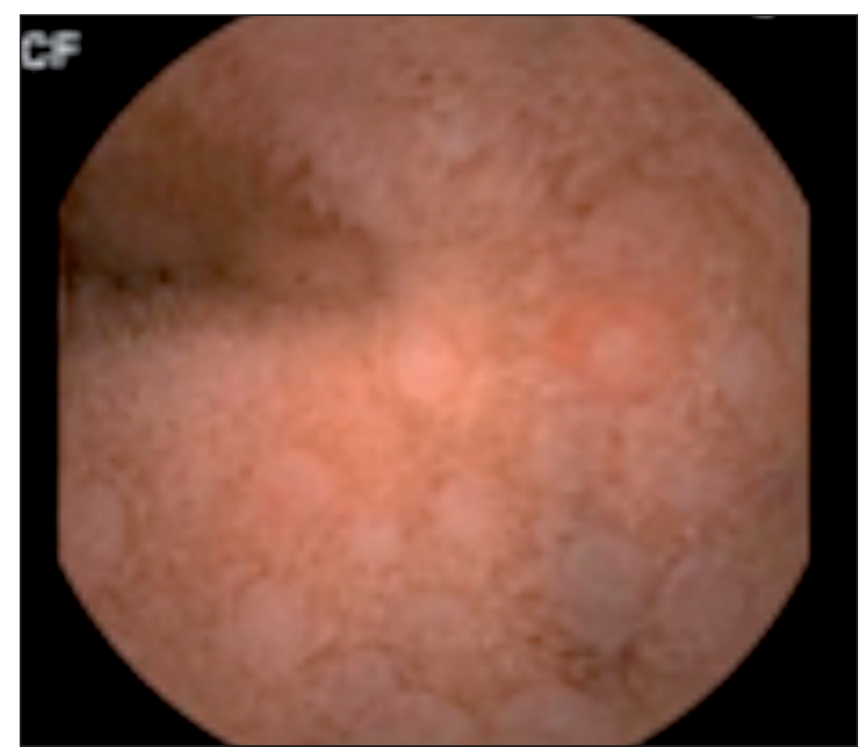

Fig. 2. Polypoid lesions seen by video capsule endosocopy, many of them just small plain nodules, in proximal portions of the small bowel.

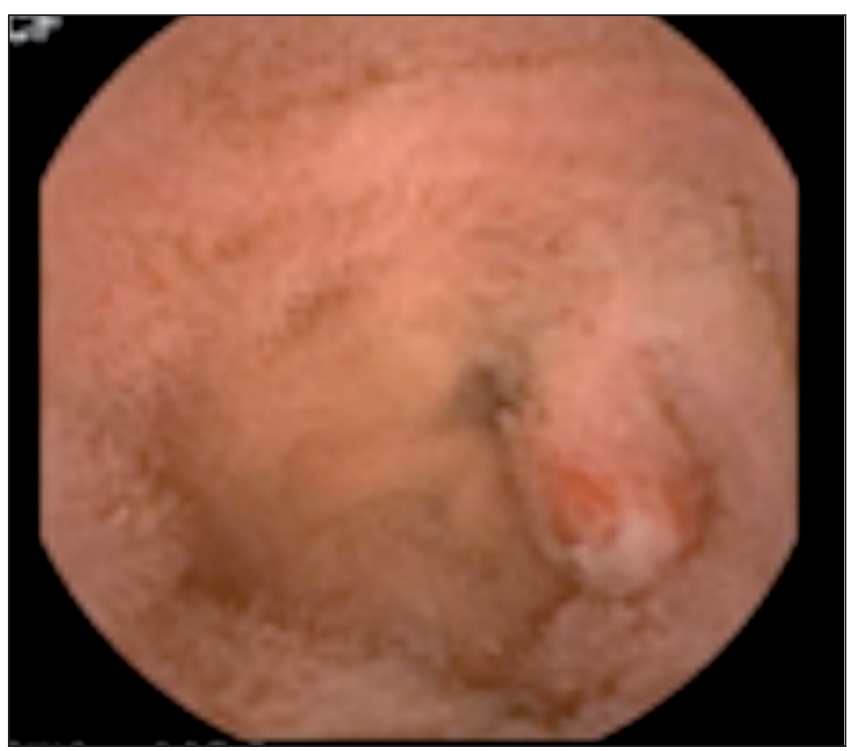

Fig. 3. In the distal small bowel, the lesions are bigger and some of them show mucosal ulcerations.

\section{REFERENCES}

1. Esaki M, Matsumoto T, Nakamura S, Suekane H, Ohji Y, Yao T, et al. Capsule endoscopy findings in intestinal follicular lymphoma. Endoscopy 2007;39:E867.

2. Akamatsu T, Kaneko Y, Ota H, Miyabayashi H, Arakura N, Tanaka E. Usefulness of double balloon enteroscopy and video capsule endoscopy for the diagnosis and management of primary follicular lymphoma of the gastrointestinal tract in its early stages. Dig Endosc 2010;22:33-8.

3. Sapoznikov B, Morgenstern S, Raanani P, Aviram A, Rabizadeh E, Prokocimer M, et al. Follicular lymphoma with extensive gastrointestinal tract involvement: Follow-up by capsule endoscopy. Dig Dis Sci 2007;52:1031-5.

4. Van Deursen CT, Goedhard JG, Jie KS, Theunissen P. Primary intestinal follicular lymphoma diagnosed by VCE and DBE. Endoscopy 2008;40(Supl. 2):E8-9. 